\title{
A new, tenth subunit of TFIIH is responsible for the DNA repair syndrome trichothiodystrophy group A
}

\author{
Giuseppina Giglia-Mari ${ }^{1}$, Frederic Coin ${ }^{2}$, Jeffrey A Ranish ${ }^{3}$, Deborah Hoogstraten ${ }^{1}$, Arjan Theil ${ }^{1}$, Nils Wijgers ${ }^{1}$, \\ Nicolaas G J Jaspers ${ }^{1}$, Anja Raams ${ }^{1}$, Manuela Argentini ${ }^{2}$, P J van der Spek ${ }^{4}$, Elena Botta ${ }^{5}$, Miria Stefanini ${ }^{5}$, \\ Jean-Marc Egly ${ }^{2}$, Ruedi Aebersold ${ }^{3}$, Jan H J Hoeijmakers ${ }^{1} \&$ Wim Vermeulen ${ }^{1}$
}

DNA repair-deficient trichothiodystrophy (TTD) results from mutations in the XPD and XPB subunits of the DNA repair and transcription factor TFIIH. In a third form of DNA repair-deficient TTD, called group $A$, none of the nine subunits encoding TFIIH carried mutations; instead, the steady-state level of the entire complex was severely reduced ${ }^{1}$. A new, tenth TFIIH subunit (TFB5) was recently identified in yeast ${ }^{2}$. Here, we describe the identification of the human TFB5 ortholog and its association with human TFIIH. Microinjection of CDNA encoding TFB5 (GTF2H5, also called TTDA) corrected the DNA-repair defect of TTD-A cells, and we identified three functional inactivating mutations in this gene in three unrelated families with TTD-A. The GTF2H5 gene product has a role in regulating the level of TFIIH. The identification of a new evolutionarily conserved subunit of TFIIH implicated in TTD-A provides insight into TFIIH function in transcription, DNA repair and human disease.

Hereditary mutations in the repair and transcription factor TFIIH are associated with three photo-hypersensitive syndromes: xeroderma pigmentosum, xeroderma pigmentosum combined with Cockayne syndrome (a neurodevelopmental disorder) and the Cockayne syndrome-like brittle-hair disease $\mathrm{TTD}^{3,4}$ (Table 1). TFIIH has a central role in transcription of RNA polymerase I and II, nucleotide excision

Table 1 Clinical and cellular features

\begin{tabular}{|c|c|c|c|c|c|c|c|c|c|}
\hline \multirow[b]{2}{*}{ Gene (s) } & \multirow[b]{2}{*}{$\begin{array}{c}\text { ХPa } \\
X P A, X P C, X P E, E R C C 2, \\
E R C C 3, E R C C 4, E R C C 5\end{array}$} & \multicolumn{3}{|c|}{ General disease characteristics } & & \multirow[b]{2}{*}{$\begin{array}{c}\text { COFS }^{\mathbf{f}} \\
E R C C 2, E R C C 5 \\
\text { ERCC6 }\end{array}$} & \multicolumn{3}{|c|}{ Individuals in this study } \\
\hline & & $\begin{array}{c}\mathbf{C S}^{\mathbf{b}} \\
C K N 1 \\
\text { ERCC6 }\end{array}$ & $\begin{array}{c}\mathbf{x P} / \mathbf{C S}^{\mathbf{c}} \\
E R C C 2, E R C C 3, \\
E R C C 5\end{array}$ & $\begin{array}{c}\text { TTD }^{\mathbf{d}} \\
\text { ERCC2 } \\
(E R C C 3)\end{array}$ & $\begin{array}{l}\text { XP/TTDe } \\
\text { ERCC2 }\end{array}$ & & $\begin{array}{l}\text { TTD1BRg } \\
\text { GTF2H5 }\end{array}$ & $\begin{array}{l}\text { TTD99RO } \\
\text { GTF2H5 }\end{array}$ & $\begin{array}{r}\text { TTD13/14PV } \\
\text { GTF2H5 }\end{array}$ \\
\hline \multicolumn{10}{|l|}{ Cutaneous symptoms } \\
\hline Photosensitive skin & Mild-severe & Mild & Moderate-severe & Mild-moderate & Severe & Severe & Mild & Very mild & Mild \\
\hline Skin cancer & Mild-severe & - & Mild-severe & - & Mild & - & - & - & - \\
\hline Brittle low-sulfur hair & - & - & - & + & $+/-$ & - & + & + & + \\
\hline Other ${ }^{\mathrm{h}}$ & $a, b$ & - & $a, b$ & $c, d$ & $b, d$ & $d$ & $\mathrm{c}-\mathrm{e}$ & $d$ & \\
\hline CS-like symptoms ${ }^{i}$ & - & ++ & + & + & - & +++ & $f, g, i, j$ & $f-k$ & $g, i, j$ \\
\hline \multicolumn{10}{|l|}{ Cellular features } \\
\hline GG-NER activity (UDS) ${ }^{j}$ & $0-70$ & 100 & $0-40$ & $10-45$ & $0-30$ & $<5$ & $15-25$ & 20 & 10 \\
\hline $\begin{array}{l}\text { Recovery of transcription } \\
\text { after UV (TC-NER) }\end{array}$ & Low-normalk & Low & Low & Low & Low & Low & Low & Low & Low \\
\hline Overall UV sensitivity & $1.5-10 \times$ & $2.5-5 x$ & $4-10 x$ & $1.5-5 x$ & $2.5-10 \times$ & $10 x$ & $2.1 \times$ & $2.7 \times$ & $3.5 x$ \\
\hline TFIIH levelj & $60-100$ & 100 & 75 & $30-50$ & ND & ND & $\sim 30$ & $\sim 30$ & $\sim 30$ \\
\hline
\end{tabular}

aXeroderma pigmentosum. ${ }^{b}$ Cockayne syndrome. ${ }^{c}$ Combined features of xeroderma pigmentosum and Cockayne syndrome. ${ }^{d}$ Photosensitive TTD. ${ }^{e}$ Combined features of xeroderma pigmentosum and TTD ${ }^{9}{ }^{f}$ Severe features of Cockayne syndrome ${ }^{12}$. gRef. 13 . ha, actinic keratosis; b, freckling; c, collodion baby; d, ichthyosis; e, eczema. iCS, Cockayne syndrome; $f$, cataracts; g, developmental delay; h, deafness; i, mental retardation; j, short stature; $\mathrm{k}$, caries. jPercent of normal. kLow for XPA, ERCC2, ERCC3, ERCC4 and ERCC5; normal for XPC and XPE.

${ }^{1}$ Department of Genetics, Medical Genetic Cluster, Erasmus MC, P.O. Box 1738, 3000 DR Rotterdam, The Netherlands. ${ }^{2}$ Institut de Génétique et de Biologie Moléculaire et Cellulaire, CNRS/INSERM/ULP, IIIkirch, France. ${ }^{3}$ Institute for Systems Biology, Seattle, Washington, USA. ${ }^{4}$ Department of Bioinformatics, Erasmus MC, P.O. Box 1738, 3000 DR Rotterdam, The Netherlands. ${ }^{5}$ Istituto di Genetica Biochimica ed Evoluzionistica, CNR, Pavia, Italy. Correspondence should be addressed to W.V. (w.vermeulen@erasmusmc.nl).

Published online 27 June 2004; doi:10.1038/ng1387 
repair (NER) and transcription-coupled repair (TCR $)^{5}$. NER removes a wide range of DNA helix-distorting injuries, including ultraviolet light (UV)-induced lesions ${ }^{6}$. TCR is a repair pathway that eliminates both classical NER lesions and oxidative DNA injuries from the transcribed strand ${ }^{7,8}$. Photosensitive features of xeroderma pigmentosum are caused by defective NER, whereas features of Cockayne syndrome and TTD are attributed to defective TCR and transcription function. Most individuals with these syndromes associated with TFIIH carry mutations of XPD or, in a few cases, of XPB, the two TFIIH helicase subunits $^{9-12}$. A third, unidentified gene that causes photosensitive $\mathrm{TTD}^{13,14}$, called GTF2H5, also shows association with TFIIH ${ }^{1}$.

Table 2 TFIIH subunits

\begin{tabular}{|c|c|c|c|c|c|}
\hline Subunit & Mammalian & Yeast & $\begin{array}{l}\text { Predicted protein } \\
\text { size in kDa } \\
\text { (human) }\end{array}$ & Properties & $\begin{array}{l}\text { Percent identity } \\
\text { etween humans } \\
\text { and yeast }\end{array}$ \\
\hline 1 & XPB & RAD25/SSL2 & 89 & Helicase, $5^{\prime}-3^{\prime}$ & 49 \\
\hline 2 & $\mathrm{XPD}$ & RAD3 & 80 & Helicase, $3^{\prime}-5^{\prime}$ & 51 \\
\hline 3 & GTF2H1 (TFB1) & TFB1 & 62 & - & 21 \\
\hline 4 & GTF2H2 & SSL1 & 44 & Zn finger & 34 \\
\hline 5 & GTF2H3 (TFB4) & TFB4 & 34 & Ring finger & 29 \\
\hline 6 & GTF2H4 (TFB2) & TFB2 & 52 & - & 34 \\
\hline 7 & MAT1 (TFB3) & TFB3 & 32 & Ring finger & 31 \\
\hline 8 & CDK7 & KIN28 & 41 & CTD kinase & 41 \\
\hline 9 & CyclinH & CCL1 & 38 & Cyclin motifs & 23 \\
\hline 10 & TFB5 & TFB5 & 8 & Stabilization of the complex & 28 \\
\hline
\end{tabular}

Subunit 10 was identified by ref. 2 .
In Table 1 we summarize the clinical and cel-

lular features of all known cases of TTD-A, including two families recently diagnosed with TTD-A. Cells from individuals with TTD-A are only mildly UV-sensitive despite having low UV-induced DNA repair synthesis (UDS).

We previously found that treatment with highly purified TFIIH corrected the NER defect of cells from individuals with TTD-A, but none of the nine genes encoding TFIIH were mutated in these individuals ${ }^{1}$. Moreover, TFIIH isolated from cells of individuals with TTD-A had normal in vitro enzymatic activities and transiently restored the NER defect in these cells, suggesting that TFIIH was qualitatively not or only mildly defective. The total cellular TFIIH content of cells from individuals with TTD-A seemed to be very low ${ }^{1}$. a
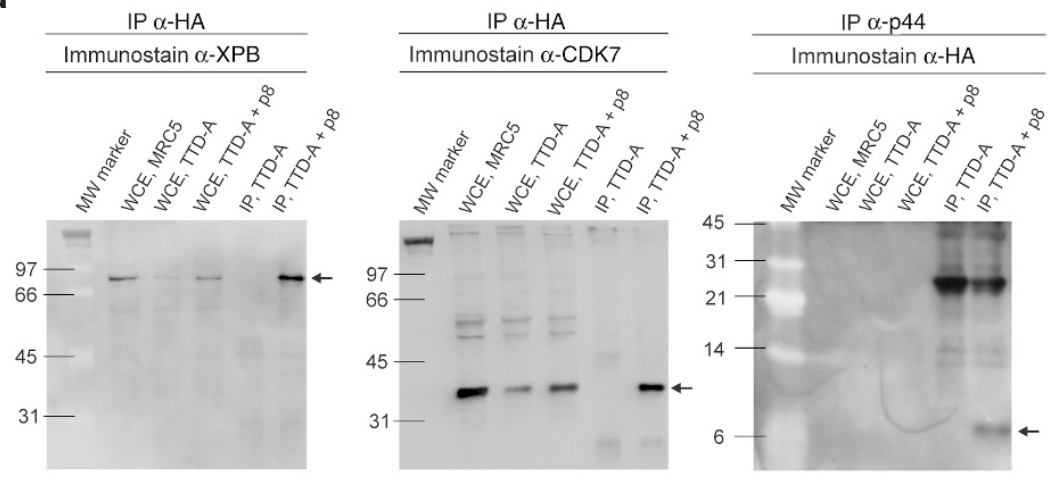

b

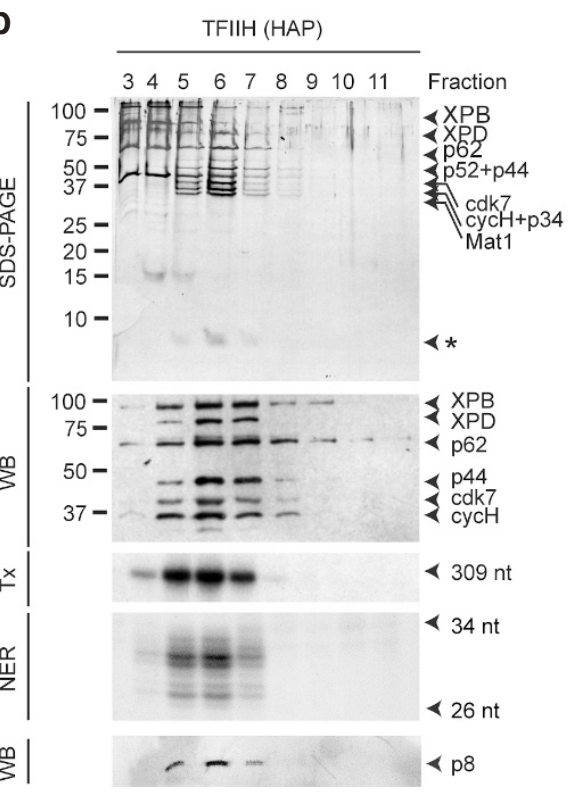

Figure 1 TFB5 is part of the TFIIH complex. (a) Immunoprecipitation (IP) assays. Lane 1, molecular weight (MW) marker; lanes 2-4: whole-cell extracts (WCE) from MRC5SV (MRC5; wild-type), TTD-A and TTD-A + p8 cells; lanes $5-6$, immunoprecipitation from TTD-A and TTD-A + p8 cell extracts. Right panel, SDS-PAGE gel separation of $10 \mu \mathrm{g}$ of proteins from whole-cell extracts and the immunoprecipitations with p44 of extracts from TTD-A and TTD-A + p8 cells. The blot was immunostained with antibody to $\mathrm{HA}$. The arrow shows the immunostained band corresponding to p8-HA. Middle and left panels, SDS-PAGE gel separation of wholecell extracts and the immunoprecipitations with antibody to HA of extracts from TTD-A and TTD-A + p8 cells. The blots were immunostained with antibodies to XPB and cdk7, respectively; the arrows show the corresponding immunostained proteins. (b) p8 is copurified with the other nine identified components of TFIIH (silver staining and western blotting; WB) and with TFIIH transcription (Tx) and repair (NER) activities. Lower panel, a rabbit polyclonal antibody raised against the $\mathrm{C}$-terminal end of $\mathrm{p} 8$ was used to detect $\mathrm{p} 8$ in the hydroxyapatite (HAP) fractions containing TFIIH. (c) MALDI-MS identification of $\mathrm{p} 8$. Upper panel, experimentally determined values and the corresponding theoretical masses of $p 8$ tryptic peptides are listed. Mass accuracy (ppm) and amino acid sequences are also shown for each peptide. Lower panel, schematic representation of MALDI peptide fingerprinting results. p8 was identified as CG14037 in the National Center for Biotechnology Information database. 
Figure 2 Correction of the DNA-repair deficiency of TTD-A by GTF2H5. (a) Microinjection of an expression vector containing GTF2H5 cDNA in TTD99R0 (TTD-A) primary fibroblasts. Micrographs show the effect of $p 8$ expression on DNA repair activity. Injected polynucleated primary fibroblasts (2), indicated by the arrow, had a high (wild-type) level of UDS, as apparent from the number of silver grains above their nuclei, relative to the uninjected surrounding fibroblasts from individuals with TTD-A (1), which had low residual UDS levels typical for TTD-A. UDS correction by $\mathrm{p} 8$ microinjection was also observed when injected into the other cells from individuals with TTD-A, TTD1BR and TTD13PV (data not shown). (b) Mutations found in the four cell lines analyzed derived from three unrelated individuals with TTD-A. The mutated base in the codon is shown in bold.

The reduced steady-state levels of TFIIH are probably a result of complex fragility due to a mutation in an unknown gene ${ }^{15}$.

A quantitative proteomic screening of promoter-associated RNA polymerase II preinitiation complexes from yeast nuclear extracts identified a new, very small protein of 72 amino acids $(\sim 8 \mathrm{kDa})$. This protein, called TFB5 (ref. 2), is a core component of yeast TFIIH. It was overlooked in previous analyses of TFIIH because of its exceptionally small size.

Database screening identified several orthologs of TFB5 (Supplementary Fig. 1 online), including a presumed human homolog, which consists of 71 amino acids and has a predicted molecular weight of $8 \mathrm{kDa}$ (referred to as $\mathrm{p} 8$ ). TFB5 was highly conserved, with a sequence identity of $28 \%$ and $56 \%$ similarity between human and yeast (Table 2). The strong evolutionary conservation of each TFIIH subunit (Table 2), in combination with the overall structural homology of the complex ${ }^{16,17}$ and the fact that the recently identified Chlamydomonas reinhardtii TFB5 ortholog (REX1S) is involved in NER ${ }^{18}$, prompted us to investigate whether $\mathrm{p} 8$ (the human TFB5 ortholog) is also part of mammalian TFIIH and whether it is the factor mutated in TTD-A.

We cloned the cDNA encoding $\mathrm{p} 8$ from primary fibroblasts. We expressed a hemagglutinin (HA)-tagged version of $\mathrm{p} 8$ in fibroblasts from individuals with TTD-A and immunoprecipitated it with antibody to HA (Fig. 1a). Both the XPB core TFIIH and the associated CAK component (CDK7) coprecipitated with p8-HA, in contrast to replication-repair factor RPA1 (data not shown). Conversely, antibody to p44 (another core TFIIH component) coprecipitated p8-HA. We conclude that, as in yeast, this small polypeptide is associated with TFIIH in mammalian cells. a

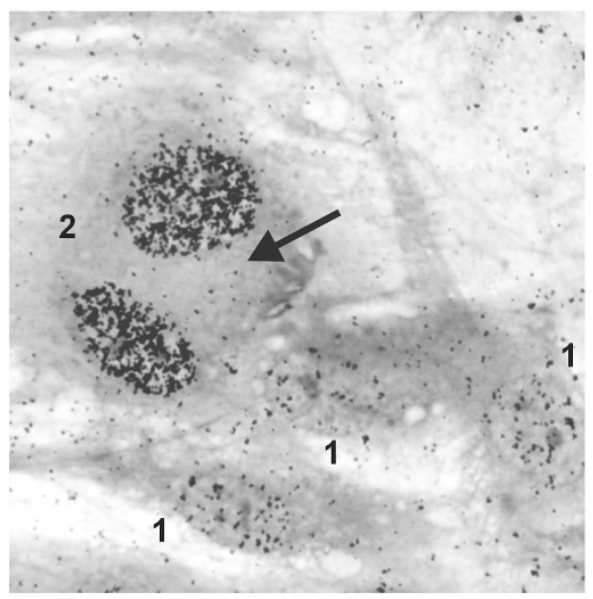

b

\begin{tabular}{|l||l|l|l|}
\hline Cell line & $\begin{array}{l}\text { Type of } \\
\text { mutation }\end{array}$ & $\begin{array}{l}\text { Amino acid } \\
\text { position }\end{array}$ & $\begin{array}{l}\text { Amino acid } \\
\text { substitution }\end{array}$ \\
\hline TTD99RO & CGA $\rightarrow$ TGA & 56 & Arg $\rightarrow$ Stop \\
\hline TTD1BR & $\begin{array}{l}\text { CGA } \rightarrow \text { TGA } \\
\text { CTG } \rightarrow \text { CCG }\end{array}$ & $\begin{array}{l}56 \\
21\end{array}$ & $\begin{array}{l}\text { Arg } \rightarrow \text { Stop } \\
\text { Leu } \rightarrow \text { Pro }\end{array}$ \\
\hline TTD13/14 PV & ATG $\rightarrow$ ACG & 1 & $\begin{array}{l}\text { Met } \rightarrow \text { Thr } \\
\text { (no start) }\end{array}$ \\
\hline
\end{tabular}

We further analyzed the association of $\mathrm{p} 8$ with TFIIH using purified TFIIH from HeLa cells ${ }^{19}$. Silver staining showed that a protein band of $\sim 8 \mathrm{kDa}$ copurified with the other TFIIH subunits and with the transcription and DNA repair activities (Fig. 1b). MALDI peptide mass fingerprint analysis on tryptic digests of this $\sim 8$-kDa band showed that all identified peptides were part of the human ortholog of TFB5 (Fig. 1c). Finally, immunoblotting using a polyclonal antibody raised against this 8-kDa polypeptide showed that it copurified with TFIIH (Fig. 1b). These experiments unambiguously identify this polypeptide (p8) as the human ortholog of TFB5 and as a new, tenth component of TFIIH.

We microinjected the cDNA into polynucleated fibroblasts from individuals with TTD-A and determined the DNA repair capacity of the injected cells by measuring UDS. Injected cells had greater UDS a

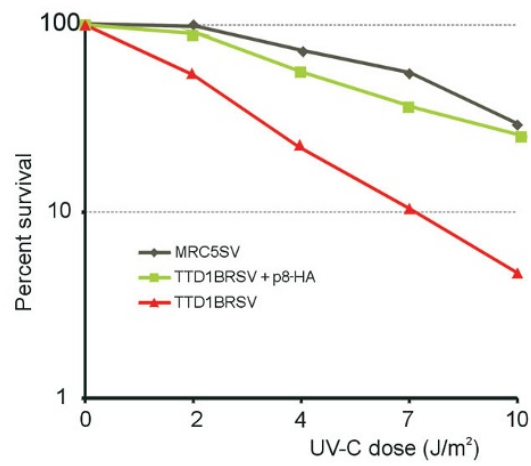

b

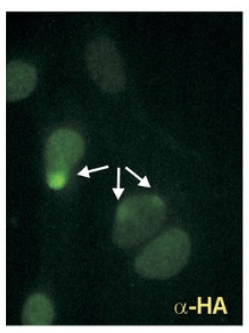

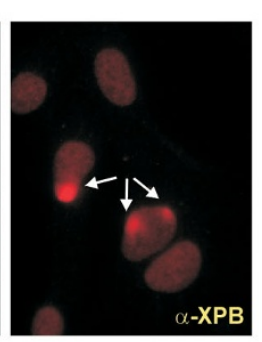

C

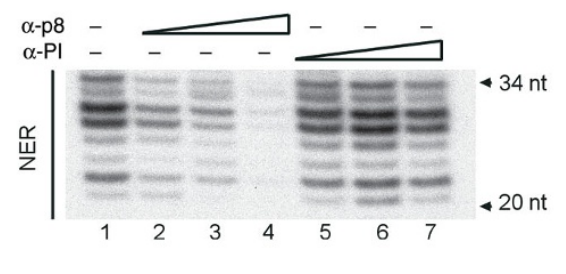

Figure 3 NER characteristics of TFB5. (a) UV survival using wild-type MRC5SV cells (black diamonds), TTD1BRSV cells (red triangles) and TTD1BRSV cells expressing TFB5-HA (green squares). The percentage of surviving cells is plotted against the UV-C dose. (b) Locally UV-irradiated (40 J m-2) TTD1BRSV cells expressing TFB5-HA were stained with antibodies to HA (left panel) and XPB (right panel). Locally damaged areas are indicated by the arrows. (c) TFIIH hydroxyapatite fraction $6(1 \mu \mathrm{l})$ was preincubated with no antibody (lane 1), with increasing amounts of antibody to p8 $(1,5$ and $10 \mu \mathrm{g}$; lanes 2-4, respectively) or with antibody control ( $\alpha-\mathrm{PI} ; 1,5$ and $10 \mu \mathrm{g}$; lanes $5-7$, respectively) before being supplemented with all other NER factors (XPC-R23b, XPA, RPA, XPG and ERCC1-XPF) in addition to damaged DNA to measure dual incision. 
(elevated grain number) than uninjected neighboring cells (Fig. 2a). GTF2H5 cDNA corrected the repair defect of cells from individuals with TTD-A to a level comparable to that observed in wild-type cells assayed in parallel, suggesting that GTF2H5 is mutated in TTD-A.

To verify that GTF2H5 is implicated in TTD-A, we analyzed genomic DNA of three unrelated individuals with TTD-A (Table 1). Each of these individuals had a different inactivating mutation in GTF2H5 (Fig. 2b). Individual TTD99RO carries a homozygous $\mathrm{C} \rightarrow \mathrm{T}$ transition at codon 56, converting CGA (arginine) into a TGA stop codon and truncating the protein by $23 \%$. Siblings TTD13PV and TTD14PV carry (homozygously) a mutation in the start codon, converting ATG to ACG. This mutation will result in either a complete loss of protein synthesis or the production of an N-terminally truncated polypeptide (lacking the first and most conserved 15 amino acids; $21 \%$ ) when a downstream AUG at codon 16 is used. Because only one allele was detected, both mutations can be considered functionally homozygous. Finally, individual TTD1BR seemed to be a compound heterozygote, with one allele identical to that of individual TTD99RO and the other allele carrying a $\mathrm{T} \rightarrow \mathrm{C}$ transition at codon 21 , converting the conserved leucine residue to a proline. Genomic mutations were also identified in isolated mRNA, as shown by RT-PCR, indicating that these alleles are expressed (data not shown). Taken together, our data unambigously identified GTF2H5 as the gene causing the NER defect in TTD-A.

To further explore the role of TFB5 in NER, we generated TTD1BRSV cells stably expressing HA-tagged TFB5. Exposure of these cells to UV-C light (Fig. 3a) showed that the UV sensitivity of the cells was comparable to that of NER-proficient cells assayed in parallel.

Using an assay in which UV damage is locally inflicted in cell nuclei by irradiation through a filter that contains pores $(5 \mu \mathrm{m})$, we previously showed that TFIIH components transiently accumulate at these sites $^{20-22}$. We applied the same procedure to cells expressing TFB5-HA to test the participation of TFB5 in the NER reaction in vivo. At local damaged sites, marked by the accumulation of endogenous XPB, TFB5-HA also accumulated (Fig. 3b). This indicates that TFB5 participates in the NER reaction, like other NER factors ${ }^{21-23}$.

We further tested the involvement of TFB5 in NER in a reconstituted in vitro incision-excision repair assay, using recombinant NER factors, purified TFIIH and a plasmid containing a cisplatin adduct. Addition of increasing amounts of purified polyclonal antibodies to TFB5 incubated with a fixed amount of purified TFIIH inhibited the repair reaction, whereas treatment with preimmune serum had no effect (Fig. 3c). These results indicate that TFB5 participates in NER as a part of TFIIH.

The NER defect and TTD-specific features in TTD-A seem to be linked to a reduced steady-state level of the total amount of TFIIH ${ }^{1}$. Cells that express TFB5-HA have a concomitantly higher XPB level (Fig. 4a), suggesting that TFB5 has a stabilizing function and protects TFIIH from degradation. Similar observations were made in cells stained with antibodies directed against other TFIIH components, namely p62, p44 and CDK7 (data not shown), indicating that the intranuclear levels of the entire TFIIH complex were increased. In contrast, levels of non-TFIIH NER factors (ERCC1) were not increased (Fig. 4b). Additionally, in immunoblot experiments (Fig. 1a), elevated levels of XPB and CDK7 were observed after expression of TFB5 expression. These experiments suggest that TFIIH concentration depends on the presence of TFB5. We transiently overexpressed TFB5HA in MRC5 cells (wild-type SV40-immortalized). Even in these TFB5-proficient cells, transient overexpression of TFB5 seemed to increase the cellular TFIIH content (Fig. 4c), suggesting that expression of TFB5 regulates TFIIH concentration in vivo.

One of the most obvious cellular phenotypes of cultured cells from individuals with TTD-A is the severely reduced level $(\sim 30 \%)$ of TFIIH (Table 1). The fact that TFB5 transiently increases the level of TFIIH, even in wild-type cells (Fig. 4c), indicates that this 8 -kDa protein is involved in regulating steady-state levels of TFIIH. Because mRNA levels of TFIIH components are not reduced in cells from individuals with TTD-A ${ }^{1}$, control of the amounts of TFIIH by TFB5 must be posttranscriptional. Regulation at the translational or post-translational level is suggestive of a chaperone-like function for TFB5 in complex assembly or maintenance. An altered tertiary structure of TFIIH, by the absence of TFB5, might impede both the longevity of this complex and its repair and transcription activity.

Although parallels of TFIIH composition, structure and function (Table 2) can be drawn between humans and yeast, differences in the relative contribution of TFB5 to transcription efficiency are also observed. In yeast, the absence of TFB5 seemed to affect the efficiency of transcription and NER, whereas in humans, mutations of TFB5 do not seriously affect transcription ${ }^{24}$. Furthermore, in humans the absence of TFB5 seemed to predominantly affect TFIIH stability and steady-state levels, whereas in yeast, absence of TFB5 did not reduce

a

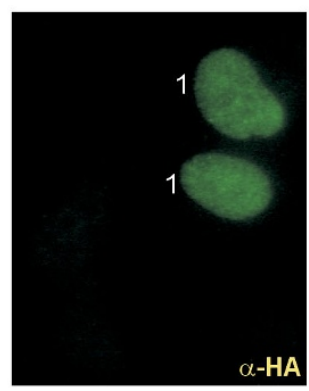

b

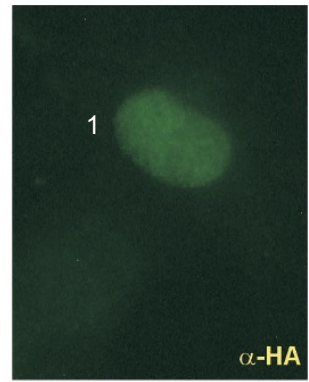

C

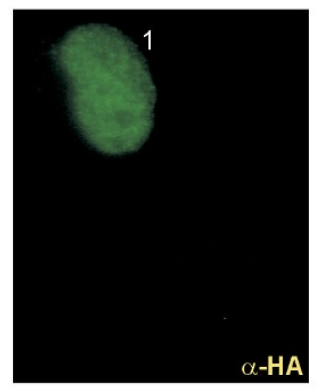

Figure 4 Recovery of reduced concentrations of TFIIH in fibroblasts from individuals with TTD-A expressing TFB5-HA. (a) Mass population of TTD1BRSV cells expressing TFB5-HA. Cells expressing TFB5-HA (left panel) had an increased level of XPB (right panel). (b) Mass population of TTD1BRSV cells expressing TFB5-HA. ERCC1 levels (right panel) were not influenced by expression of TFB5-HA (left panel). (c) MRC5SV cells expressing TFB5-HA (left panel) showed an increased level of XPB (right panel) 1, cells expressing TFB5-HA. 
TFIIH levels, as judged by levels of TFB1 and Kin28 (J.A.R., unpublished data). Future experiments should identify the mechanistic details of TFB5 function in humans and yeast.

The severe effect of GTF2H5 mutations on NER function suggests that NER requires higher concentrations of TFIIH than does transcription. Live cell studies showed that TFIIH participates substantially longer in NER than in transcription ${ }^{22}$, providing a possible explanation for the increased need for sufficient amounts of TFIIH in NER. Additionally, an altered structure of TFIIH caused by mutant TFB5 may primarily affect NER function.

In conclusion, the identification of TFB5 as a new, evolutionarily conserved, tenth subunit of TFIIH provides essential information to understand the molecular mechanism of TFIIH function in DNA repair, transcription and human disease. These findings unambiguously identify GTF2H5 as the gene causing the NER defect in TTD-A and provide an opportunity to begin to dissect the molecular details underlying the disease.

\section{METHODS}

Cell lines, culture conditions and transfections. We cultured primary human fibroblasts TTD99RO, TTD1BR, TTD13PV, TTD14PV (all TTD-A) and C5RO (wild-type, NER-proficient) under standard conditions at $37^{\circ} \mathrm{C}, 5 \% \mathrm{CO}_{2}$ and $3 \%$ oxygen in Ham's F-10 medium supplemented with $12 \%$ fetal calf serum and antibiotics (penicillin and streptomycin). We cultured SV-40 transformed cell lines TTD1BR-SV (TTD-A) and MRC5-SV (wild-type) at $37^{\circ} \mathrm{C}, 5 \% \mathrm{CO}_{2}$ in a 1:1 mixture of Ham's F-10 medium and Dulbecco's modified Eagle medium (Gibco) supplemented with $8 \%$ fetal calf serum and antibiotics. We cloned C-terminally HA-tagged TFB5 into the expression vector pCDNA3 (Invitrogen) and transfected this construct into TTD1BRSV fibroblasts using FUGENE 6 transfection reagent (Roche). We subjected transfectants to triple UV-C irradiation of $8 \mathrm{~J} \mathrm{~m}^{-2}$ with 2-d intervals to enrich the population of cells expression TFB5-HA. One week after the last irradiation, stable transfectants were single cell-sorted (FACSVantage, Becton Dickinson). We tested colonies derived from single cells by immunofluorescence with antibody to HA after expressing the fusion gene. We tested the repair capacity of a selected clone stably expressing TFB5-HA (UV survival).

Immunoprecipitation and immunoblot analysis. We prepared whole-cell extracts from MRC5SV, TTD1BRSV and TTD1BRSV+TFB5HA fibroblasts by isolating cells from six petri dishes $(13-\mathrm{cm})$ per cell line. We collected the cell pellets and washed them once in phosphate-buffered saline (PBS) before lysing them by douncing ( 20 strokes using a 12.61-mm dounce homogenizer, Bellco Glass) in $2 \mathrm{ml}$ of buffer A (50 mM Tris (pH 7.9), $150 \mathrm{mM} \mathrm{NaCl}, 20 \%$ glycerol, $0.1 \%$ Nonidet-P40 and $5 \mathrm{mM} \beta$-mercaptoethanol) supplemented with antiproteases. We incubated cellular extracts from MRC5SV, TTD1BRSV and TTD1BRSV+TFB5HA fibroblasts overnight at $4{ }^{\circ} \mathrm{C}$ in buffer A with antibodies to HA (12CA5) and p44 (1H5) cross-linked to protein A-sepharose beads (Amersham Biosciences). Before immunoprecipitations, we washed crosslinked beads three times in buffer A. After incubation with the extracts, we washed the beads extensively with buffer A and then analyzed them by SDSPAGE and immunoblotted them using antibodies to XPB (1B3), cdk7 (2F8) and HA (3F10).

Mass spectrometry. We separated TFIIH subunits on a $15 \%$ polyacrylamide SDS-PAGE gel and stained them with silver ${ }^{25}$. The band migrating below the $10-\mathrm{kDa}$ marker was excised and digested in the gel with trypsin ${ }^{26}$. We concentrated the peptide extracts by speed-vac, purified them by chromatography on a C18 reverse-phase 'ZipTip' pipette tip (Millipore) and finally eluted them in $2 \mu \mathrm{l}$ of $50 \%$ acetonitrile. We mixed $0.5 \mu \mathrm{l}$ of peptides either with an equal volume of saturated alpha-cyano- 4 hydroxycinnamic acid (dissolved in 50\% acetonitrile) or with the same volume of saturated 2,5-dihydroxybenzoic acid (dissolved in 20\% acetonitrile). Both matrices were purchased from LaserBio Labs. We carried out mass measurements on a Bruker Reflex IV MALDI-TOF spectrometer in the positive-ion reflector mode. The mass acquisition range was $800-3,000 \mathrm{Da}$ with a low mass gate set at $700 \mathrm{Da}$. Internal calibration was done using autolytic trypsin peptides $\left(\mathrm{MH}^{+}\right.$with $\mathrm{m} / z=842.51,2211.11$ and 2807.47). Monoisotopic peptide masses were assigned manually using the Bruker X-TOF software. We carried out database searches (National Center for Biotechnology Information, proteins of human origin) with the Profound program using the following parameters: molecular weight of 0-10 kDa, trypsin digestion (one missed cleavage tolerated), cysteines modified by carbamidomethylation, methionine oxidation and mass tolerance of $75 \mathrm{ppm}$. Four of five $\mathrm{p} 8$ peptides were detected in the MALDI spectrum using both alphacyano-4 hydroxycinnamic (data not shown) and 2,5-dihydroxybenzoic acids as matrix.

Microinjection and DNA repair assays. We microinjected GTF2H5 cDNA constructs (with and without a HA tag) into fibroblasts from individuals with TTD-A (TTD99RO, TTD1BR) as described ${ }^{27}$. Three days before microinjection, we fused cells using inactivated Sendai virus and seeded them onto coverslips. To allow the expression of the injected gene, we exposed cells to $15 \mathrm{~J} \mathrm{~m}^{-2}$ UV-C $24 \mathrm{~h}$ after injection and incubated them for $2 \mathrm{~h}$ in culture medium supplemented with ${ }^{3} \mathrm{H}$-thymidine. After removing excess free ${ }^{3} \mathrm{H}$-thymidine, we fixed cells. We determined DNA repair capacity (UDS) as the amount of autoradiographic grains above the nuclei, which is usually severely reduced in NER-deficient cells (for details see ref. 1). We determined the UV sensitivity of TTD1BRSV (TTD-A), TTD1BRSV+TFB5-HA (TTD-A corrected) and NERproficient MRC5SV (wild-type) human fibroblasts by incorporation of ${ }^{3} \mathrm{H}$ thymidine, as described previously ${ }^{28,29}$.

Mutation analysis of TFB5. We extracted total RNA (10 $\mu \mathrm{g})$ from wild-type fibroblasts (C5RO) and primary fibroblasts from individuals with TTD-A (TTD99RO; TTD1BR; TTD13PV and TTD14PV) using RNeasy Mini columns (Qiagen) and used it to prepare cDNA by reverse transcription. Reverse transcription was carried out using the SuperScript First-Strand Synthesis System for RT-PCR (Invitrogen). We amplified GTF2H5 cDNA using puReTaq ReadyTo-Go PCR Beads (Amersham) and 10 pmol of primers (Tm: $60^{\circ}$ ). We amplified the coding region of genomic GTF2H5 using two sets of primers covering the coding exons 2 and 3 . Primers sequences are available on request. We amplified genomic DNA (100-500 ng) using puReTaq Ready-To-Go PCR beads (Amersham) and $10 \mathrm{pmol}$ of each primer set. Amplification products were cloned into T-easy vector (Promega). For each cell line, we sequenced 18 independent clones using BaseClear lab services.

Immunofluorescence. Two days after micro injection or transfection, we washed cells twice in PBS, fixed them in 2\% paraformaldehyde, permeabilized them two times for $10 \mathrm{~min}$, rinsing in PBS containing $0.1 \%$ Triton X-100 (PBS$\mathrm{T})$ and then washed them with PBS plus $0.15 \%$ glycine and $0.5 \%$ bovine serum albumin (PBS+). We diluted antibodies in PBS+ and incubated cells with antibodies for $2 \mathrm{~h}$ at room temperature in a moist chamber. Dilutions were 1,000X for antibodies to XPB (IB3) and to HA (3F10, Roche). We then washed coverslips (five times for 5 min each) in PBS-T and incubated them with the secondary antibody: goat antibody to mouse conjugated with Cy3 (The Jackson Laboratory) and goat antibody to rat conjugated with Alexa 488 (Molecular Probes), respectively, each diluted 1,000 $\times$ in PBS+. After the same washing procedure, we mounted coverslips in Vectashield mounting medium (Vector Laboratories) containing $1.5 \mu \mathrm{g} \mu \mathrm{l}^{-1}$ of DAPI. We produced epifluorescent and phase-contrast images on a Leitz Aristoplan microscope equipped with epifluorescence optics and a PLANAPO 63×/1.40 oil immersion lens.

Dual incision NER assay. We carried out the dual incision assay as described $^{30}$ in $25 \mu \mathrm{l}$ of dual incision buffer supplemented with $2 \mathrm{mM}$ ATP. Each reaction contained $5 \mathrm{ng}$ of XPG, $15 \mathrm{ng}$ of ERCC1, $10 \mathrm{ng}$ of XPCHR23B, $50 \mathrm{ng}$ of RPA, $25 \mathrm{ng}$ of XPA and TFIIH as indicated. After $10 \mathrm{~min}$ of preincubation at $30^{\circ} \mathrm{C}$, we added $30 \mathrm{ng}$ of Cys-platinum damaged DNA and continued the reactions for $90 \mathrm{~min}$ at $30^{\circ} \mathrm{C}$. We detected the excised fragment on $14 \%$ urea-acrylamide after annealing with $9 \mathrm{ng}$ of the complementary oligonucleotide and adding four radioactively labeled dCMP $\alpha-\mathrm{P}^{32}$ $\left(3,000 \mu \mathrm{Ci} \mathrm{mmol}{ }^{-1}\right)$ residues by Sequenase V2.1 (USB). Before adding it to the repair reactions, we preincubated TFIIH with either polyclonal antibodies to $\mathrm{p} 8$ or preimmune serum. The rabbit polyclonal antibody was produced by coupling the peptide VGELMDQNAFSLTQK (57-71) to ovalbumin and injecting it in rabbits. 
Local ultraviolet irradiation. We irradiated cultured cells with UV $(254 \mathrm{~nm})$ at $40 \mathrm{~J} \mathrm{~m}^{-2}$ through an isopore polycarbonate filter (Millipore) containing $5-\mu \mathrm{m}$ pores as described ${ }^{20,21}$. After removing the filter, we fixed cells with paraformaldheyde and further processed them for immunocytochemistry as described above.

URL. The Profound program is available at http://prowl.rockefeller.edu/.

GenBank accession numbers. The complete human cDNA sequence is available under BC060317, AK055106 or AJ634743.

Note: Supplementary information is available on the Nature Genetics website.

\section{ACKNOWLEDGMENTS}

This work was supported by grants from European Molecular Biology

Organization, the Dutch Basic Research Organization, the Dutch Cancer Society, European Union and the National Institute of Health.

\section{COMPETING INTERESTS STATEMENT}

The authors declare that they have no competing financial interests.

Received 13 February; accepted 3 May 2004

Published online at http://www.nature.com/naturegenetics/

1. Vermeulen, W. et al. Sublimiting concentration of TFIIH transcription/DNA repair fac tor causes TTD-A trichothiodystrophy disorder. Nat. Genet. 26, 307-313 (2000).

2. Ranish, J.A. et al. Identification of TFB5, a new component of general transcription and DNA repair factor IIH. Nat. Genet. advance online publication, 27 June 2004 (doi:10.1038/ng1385)

3. Lehmann, A.R. The xeroderma pigmentosum group $D(X P D)$ gene: one gene, two functions, three diseases. Genes Dev. 15, 15-23 (2001).

4. Bootsma, D., Kraemer, K.H., Cleaver, J.E. \& Hoeijmakers, J.H. Nucleotide excision repair syndromes: xeroderma pigmentosum, Cockayne syndromes, and trichothiodystrophy. in The Genetic Basis of Human Cancer (B. Vogelstein and K. W. Kinzler, eds.) 245-274 (McGraw-Hill, New York, 1998).

5. Egly, J.M. The 14th Datta Lecture. TFIIH: from transcription to clinic. FEBS Lett. 498, 124-128 (2001).

6. Hoeijmakers, J.H. Genome maintenance mechanisms for preventing cancer. Nature 411, 366-374 (2001).

7. de Boer, J. et al. Premature aging in mice deficient in DNA repair and transcription. Science 296, 1276-1279 (2002).

8. Le Page, F. et al. Transcription-coupled repair of 8-oxoguanine: requirement for XPG $\mathrm{TFIIH}$, and CSB and implications for Cockayne syndrome. Cell 101, 159-171 (2000).

9. Broughton, B.C. et al. Two individuals with features of both xeroderma pigmentosum and trichothiodystrophy highlight the complexity of the clinical outcomes of muta- tions in the XPD gene. Hum. Mol. Genet. 10, 2539-2547 (2001).

10. Botta, $\mathrm{E}$ et al. Analysis of mutations in the XPD gene in Italian patients with trichothiodystrophy: site of mutation correlates with repair deficiency, but gene dosage appears to determine clinical severity. Am. J. Hum. Genet. 63, 1036-1048 (1998).

11. Weeda, G. et al. A mutation in the XPB/ERCC3 DNA repair transcription gene, associated with trichothiodystrophy. Am. J. Hum. Genet. 60, 320-329 (1997).

12. Graham, J.M. Jr. et al. Cerebro-oculo-facio-skeletal syndrome with a nucleotide excision-repair defect and a mutated XPD gene, with prenatal diagnosis in a triplet pregnancy. Am. J. Hum. Genet. 69, 291-300 (2001).

13. Stefanini, M. et al. A new nucleotide-excision-repair gene associated with the disorder trichothiodystrophy. Am. J. Hum. Genet. 53, 817-821 (1993).

14. Vermeulen, W. et al. Three unusual repair deficiencies associated with transcription factor BTF2(TFIIH): evidence for the existence of a transcription syndrome. Cold Spring Harb. Symp. Quant. Biol. 59, 317-329 (1994).

15. Petrini, J.H. When more is better. Nat. Genet. 26, 257-258 (2000).

16. Schultz, P. et al. Molecular structure of human TFIIH. Cell 102, 599-607 (2000).

17. Chang, W.H. \& Kornberg, R.D. Electron crystal structure of the transcription factor and DNA repair complex, core TFIIH. Cell 102, 609-613 (2000).

18. Cenkci, B., Petersen, J.L. \& Small, G.D. REX1, a novel gene required for DNA repair J. Biol. Chem. 278, 22574-22577 (2003).

19. Gerard, M. et al. Purification and interaction properties of the human RNA polymerase B(II) general transcription factor BTF2. J. Biol. Chem. 266, 20940-20945 (1991).

20. Mone, M.J. et al. Local UV-induced DNA damage in cell nuclei results in local transcription inhibition. EMBO Rep. 2, 1013-10117 (2001).

21. Volker, M. et al. Sequential assembly of the nucleotide excision repair factors in vivo. Mol. Cell 8, 213-224 (2001)

22. Hoogstraten, D. et al. Rapid switching of TFIIH between RNA polymerase I and II transcription and DNA repair in vivo. Mol. Cel. 10, 1163-1174 (2002).

23. Rademakers, S. et al. Xeroderma pigmentosum group A protein loads as a separate factor onto DNA lesions. Mol. Cell. Biol. 23, 5755-5767 (2003).

24. Tirode, F., Busso, D., Coin, F. \& Egly, J.M. Reconstitution of the transcription factor TFIIH: assignment of functions for the three enzymatic subunits, XPB, XPD, and cdk7. Mol. Cel/ 3, 87-95 (1999).

25. Rabilloud, T. Silver staining of 2-D electrophoresis gels. Methods Mol. Biol. 112 297-305 (1999)

26. Cavusoglu, N., Brand, M., Tora, L. \& Van Dorsselaer, A. Novel subunits of the TATA binding protein free TAFll-containing transcription complex identified by matrixassisted laser desorption/ionization-time of flight mass spectrometry following onedimensional gel electrophoresis. Proteomics 3, 217-223 (2003).

27. Vermeulen, W. et al. Clinical heterogeneity within xeroderma pigmentosum associated with mutations in the DNA repair and transcription gene ERCC3. Am. J. Hum. Genet. 54, 191-200 (1994).

28. Sijbers, A.M. et al. Mutational analysis of the human nucleotide excision repair gene ERCC1. Nucleic Acids Res. 24, 3370-3380 (1996).

29. van Gool, A.J. et al. The Cockayne syndrome $B$ protein, involved in transcription-coupled DNA repair, resides in an RNA polymerase II-containing complex. EMBO J. 16, 5955-5965 (1997)

30. Riedl, T., Hanaoka, F. \& Egly, J.M. The comings and goings of nucleotide excision repair factors on damaged DNA. EMBO J. 22, 5293-5303 (2003). 